Review Article

\title{
Management of abrasions in oral and maxillofacial surgery - a review
}

\author{
Muralee Mohan C. ${ }^{1}$, Gopinath Thilak P. S. ${ }^{2}$, Pratiksha Shetty ${ }^{3}$, Ashish J. Rai ${ }^{4}$, Saurabh M. Gohil ${ }^{5}$ \\ ${ }^{1}$ Professor, ${ }^{2,3,4}$ Assistant Professor, ${ }^{5}$ Post Graduate Student, Department of Oral \& Maxillofacial Surgery, A.B. Shetty \\ Memorial Institute of Dental Sciences, Mangalore, Karnataka, India. \\ *Corresponding Author : Saurabh M. Gohil, Post Graduate Student, Department of Oral \& Maxillofacial Surgery, A.B. Shetty Memorial \\ Institute of Dental Sciences, Nitte University, Mangalore - 575 018, Karnataka, India. \\ Mobile: 9845197263/09819929726 E-mail:dr.saurabhgohil@gmail.com
}

$\begin{array}{ll}\text { Received } & : \text { 25-04-2016 } \\ \text { Review Completed } & : \text { 17-06-2016 } \\ \text { Accepted } & : \text { 04-08-2016 }\end{array}$

Keywords : abrasions, face, hydrocolloid, dressings, maxillofacial

\begin{tabular}{|c|}
\hline Access this article online \\
\hline Quick Response Code \\
\hline
\end{tabular}

\begin{abstract}
:
Soft tissue injuries, whether isolated or in combination with other injuries, are among the most common traumatic craniofacial injuries encountered by emergency department personnel and maxillofacial surgeons. Despite this high incidence, there are very few studies that systematically investigate the management of these injuries and therefore, there is no widely accepted classification scheme or treatment algorithms to guide evaluation and treatment. As a result, most critical management decisions are left exclusively to the discretion of the treating surgeon with limited prospective or retrospective data, beyond personal experience, available to guide treatment. This may lead to many disparate approaches to both short-term and longterm management. In this review article, we give a brief account of management of facial abrasions, including tetanus prophylaxis, pain assessment, cleaning, definitive closure and dressing of facial abrasions.
\end{abstract}

\section{Introduction}

A large part of the Casualty department's workload entails the management of acute traumatic wounds. Wound care involves extensive knowledge of skin anatomy, the physiology of wound healing, and the impact of infection on healing and to provide self-care advice to patients who can manage their own wounds ${ }^{(1)}$. Wounds accounts for approximately $25-30 \%$ of the total workload of Casualty department ${ }^{(2)}$. The commonest wounds presenting are lacerations caused by blunt trauma, cuts, abrasions and burns, all of which respond rapidly to treatment and heal without complication $^{(3)}$.

A wound signifies a break in the continuity of tissues covering the body and is usually associated with a defect ${ }^{(4)}$. In traumatic and intentional injury, there is rupture of blood vessels, resulting in bleeding, followed by clot formation. The type of trauma and extent of damage serve to further classify wounds for prognosis and treatment options ${ }^{(5)}$.

\section{Abrasions}

Abrasions result from deflecting trauma, such as sliding along pavement, dirt or glass that removes the epithelial layer and the papillary layer of dermis and leaves the raw, bleeding reticular layer of the dermis exposed. This type of wound may be painful owing to exposed nerve endings in reticular dermal layer ${ }^{(6)}$.

\section{Management of Abrasions -}

There are three key elements to consider at triage

1. Accurate description of wound.

2. Documentation of mechanism of injury, tetanus immunization status, current medication and known allergies.

3. First aid measures for hemostasis, proper cleansing and 
adequate temporary closure of open wounds, which will lengthen the time available for definitive treatment.

Adequate history taking should include:

- Time lapsed since wounding.

- Circumstances/Mechanism of injury.

- First aid treatment, if any rendered.

- Tetanus immunization status.

- Allergy - hypersensitivity to adhesive tapes, dressing materials and medications.

- Current/recent medication - corticosteroid therapy, aspirin, anti-diabetic and other anti-coagulant therapy, etc.

- Social history - to ascertain whether the patient lives alone, if he or she has extended family who can assist in the managementof the wound following discharge, or the availability of primary care facilities locally.

Clostridium its Tetani is an anaerobic organism and thrives in conditions where there is devitalized tissue and hematoma formation. All patients with wounds must have their tetanus immunization status established ${ }^{(2)}$. Therefore, two elementary questions are essential when assessing the need for tetanus immunization:

- Has the patient been immunized according to Department of Health recommended guide-lines?

- Is the wound clean or tetanus-prone?

Cleansing wound essential for the prevention of infection, tattoo scarring and to exclude foreign body contaminants, such as grit $^{(1)}$. Wound infection is the most common serious complication of a simple laceration ${ }^{(7)}$.

During wounding there is a breakdown in the protective functions of the skin and micro- organisms can enter the deeper tissues and cause infection ${ }^{(8)}$. Expensive surgical dressings, tissue adhesive, strip closures, staples or sutures will not assist healing if the wound is not properly and thoroughly cleansed initially. However, Hollander et al, in a study exploring infection rates for face and scalp wounds, found that there was no difference in the outcome for patients who hadwound irrigation prior to skin closure and those who did not ${ }^{(9)}$.

\section{Pain assessment}

Pain relief should be considered prior to decontamination or cleansing of any wound. Pain assessment is an integral part of the first-line management and triage decisionmaking process ${ }^{(10)}$. A number of pain assessment tools are used in the Casualty setting; these tools help to provide the health professional with objective data that can be used to plan relevant pain-relieving measures and measure their effects. The type of analgesia and route of administration will depend on Casualty department guidelines and on individual patients' suitability for oral or intramuscular medication. The well-known visual analogue scale (VAS) and numeric rating scale (NRS) for assessment of pain intensity agree well and are equally sensitive in assessing acute pain, and they are both superior to a fourpoint verbal categorical rating scale (VRS $)^{(11)}$.

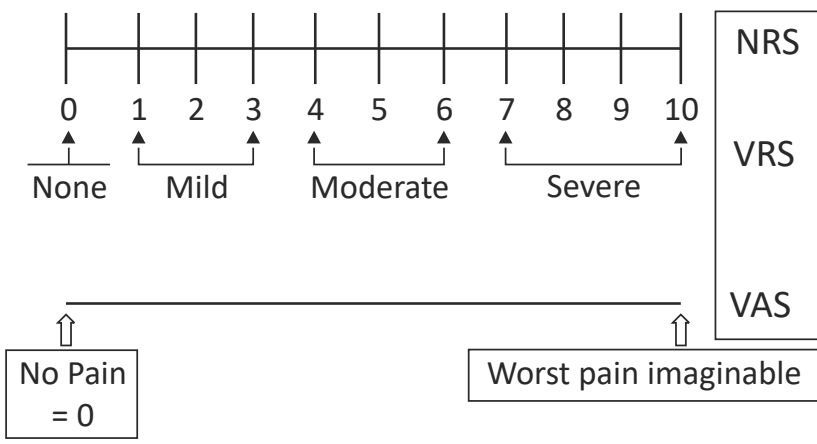

Figure 1 : Diagrammatic representation of VAS, NRS and VRS.

\section{Cleansing agent}

Irrigation of the wound is essential to remove contaminants and reduce infection rates. Topical antiseptic solutions have been discredited recently, with normal saline coming to the fore as the optimum cleanser ${ }^{(1,7,12)}$. Normal saline is favored as it is an isotonic solution and does not interfere with the normal healing process. Tap water is commonly used in the community for cleansing wounds because it is easily accessible, efficient and cost effective, however, there is an unresolved debate about its use. To date, there is no evidence that using tap water to cleanse acute wounds in adults increases infection on the contrary, some evidence suggests that it reduces $\mathrm{it}^{(13)}$. However, some recent studies have shown that ordinary tap water of drinking quality has proved as effective at irrigating and cleansing wounds as normal 
saline solution ${ }^{(7,14,15)}$.Antiseptic solutions such as cetrimide and chlorhexidine claim to destroy bacteria and have a deterrent effect on wounds. Holt reports that most of these solutions require 20 minutes contact time with bacteria to have an effect. There is little evidence to support the continued use of povidone-iodine solution as a first-line method of wound cleaning; it is not recommended for routine cleansing or prophylaxis due to its cytotoxic side effects on acute and healing wounds. Other solutions, such as hydrogen peroxide, were previously thought to have decontaminating and desloughing properties, but these properties are greatly reduced once the solution is in contact with blood and pus. Also, the thermal effect of the solution adversely affects healthy tissue ${ }^{(1)}$.

\section{Definitive Closure}

Definitive closure of a wound will depend on the information gathered from the history, assessment and evaluation of the patient. The method of closure will also depend on the patient's ability to continue his or her activities of daily living on leaving the Casualty department ${ }^{(5)}$.

If the wound extends deeply into the dermal layer, notable scarring from granulation tissue formation will result. Excision of the remaining dermal tissue or excision of secondary scar tissue, with primary closure of skin wounds with 4-0 resorbable suture in the dermal layer and 6-0 prolene sutures at the surface, is indicated. Exposure of abraded skin wound to excessive sunlight during the first six months after injury may cause permanent hyper pigmentation. These healing wounds may benefit from protection with a sun blocking agent with a sun protection factor of 15 or more ${ }^{(6)}$.

\section{Dressings}

As with skin closure products, wound dressings have proliferated and many nurses are now confronted with several products with essentially the same effect and mode of action. The process of dressing selection is determined by a number of factors that include the nature and location of the wound, the presence or risk of infection and the amount of exudate produced ${ }^{(16)}$. Superficial wounds generally produce little exudate and can be dressed with a hydrocolloid or semipermeable film dressing. Low adherent dressings are especially useful for the management of abrasions.

According to a study, basic dressings such as paraffin gauze (both plain and medicated), hydrocolloid dressings produce improved healing rates in partial thickness wounds such as burns, donor sites, superficial traumatic injuries and some types of surgical wounds. There is also a body of evidence to suggest that their use is associated with a reduction in wound pain, enhanced quality of life, (including the ability to wash or shower) and also an improvement in the quality of the healed wound ${ }^{(17)}$.A patient came to our department with huge facial abrasion involving deep dermal layers. We used medicated paraffin gauze as dressing material, which showed good result after 2 weeks. (Figure 2-4)

According to a study, Bovine amniotic membrane [Amnisite $B A(R)]$ was used as dressings for facial abrasions. This study concluded that facial abrasion can be effectively treated with dried bovine amniotic membrane with a shortening of epithelization period and similar effectiveness as previous foam dressings. In addition, dried bovine amniotic membranes have several benefits. easy appliance to any parts of face, reducing the frequency of dressing changes, early adaptation of patient to social life and so on, These presented benefits make this material a new standard for treatment of facial abrasion ${ }^{(18)}$.

According to a study, human amniotic membrane was used with patients with necrotizing cervical fasciitis. Advantages of this being, it is available at no cost especially in a center where delivery cases are done routinely, and also there is better and faster healing with minimal scar. Similarly we can use this for deep dermal abrasive defects. ${ }^{(19)}$.

To prevent drying and desiccation of exposed wound surfaces, the abrasion should be covered with a thin layer of antibiotic ointment, such as bacitracin, and dressed with cotton gauze or covered with an antibiotic coated cellulose acetate gauze ${ }^{(6)}$. 
No single dressing is suitable for the management of all types of wounds and few are suited to the treatment of a single wound during all stages of the healing cycle. Successful wound management depends on a flexible, knowledgeable approach to selection of a suitable dressing $^{(16)}$.

\section{Conclusion}

Minor wounds that commonly present to the Casualty department generally heal rapidly andpatients return to normal activity quite quickly. However, there are always exceptions to this rule and it is the casualty attendant who caninfluence the outcome of this group of patients. Adequate knowledge about wounding, wound assessment and management strategieswill improve theoutcome for a significant number of patients who just have 'a simple cut'.
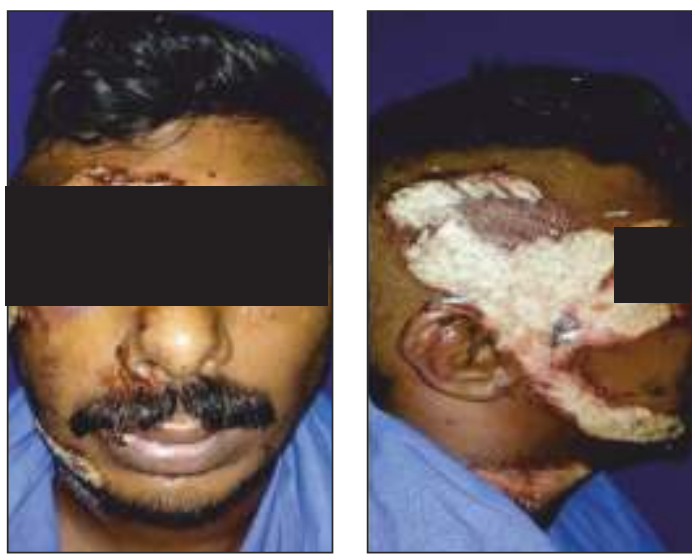

Figure 2 : Patient with Facial abrasion

References

1. Wardrope J, Smith JA. The Management of Wounds and Burns. Oxford, Oxford University Press.

2. Dealey C. The Care of Wounds: A Guide for Nurses. Second Edition. Oxford, Blackwell Science.

3. Lippert H. Compendium: Wounds and Wound Management. Heidenheim.

4. Small V. Management of cuts, abrasions and lacerations. nursing Standard. 15, 5, 41-44.

5. Moscati RM. Wound irrigation with tap water. Academic Emergency Medicine. 5, 11, 1076-1080.

6. Courtenay M, Butler M. Nurse Prescribing: Principles and Practice. London, Greenwich Medical Media.

7. Hollander JE. Irrigation in facial and scalp lacerations: does It alter outcome 7 Annals of Emergency Medicine. 31, 1,73-77.

8. Group., Manchester Triage. Emergency Triage. London, BMJ Publishing Group.

9. Walsh M. Disorders of the skin. Nurse Practitioners: Clinical Skills and Professional Issues. Oxford, Butterworth-Heinemann.

10. Angeras $\mathrm{MH}$. Comparison between sterile saline and tap water for the cleansing of acute traumatic soft tissue wounds. European Journal of Surgery 158, 6-7, 347-350.
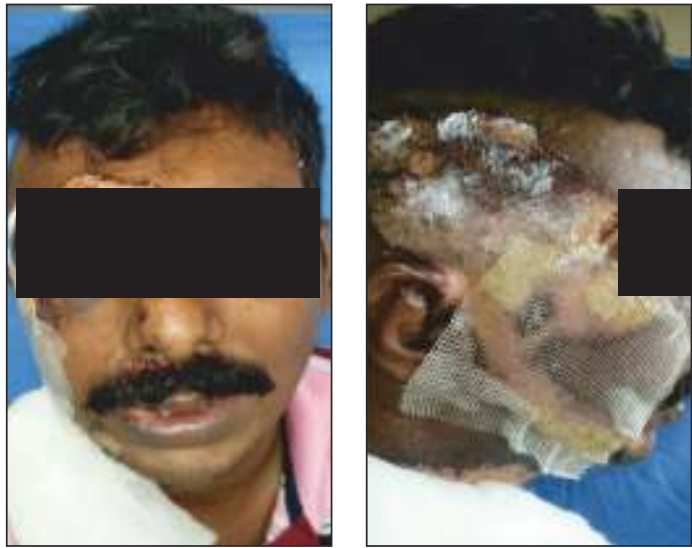

Figure 3 : Medicated paraffin dressing placed on the abrasion
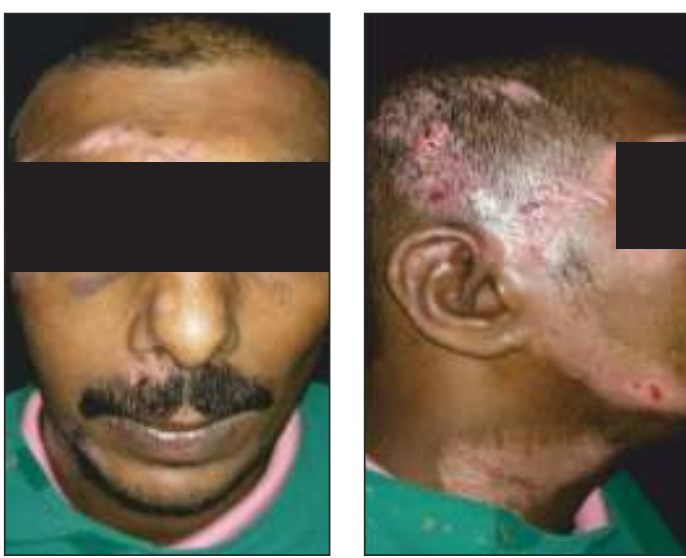

Figure 4 : Patient after 2 weeks of follow-up

11. Riyat MS, Quniton DN,. Tap water as a wound cleansing agent in A\&E. Journal of Accident \& Emergency Medicine. 14, 3, 165-166.

12. Thomas S. A Structured Approach to the Selection of Dressings. http://www.smtl.co.ukIWorld-Wide-Wounds

13. Kim J, Park J, Park N. Treatment of Facial Abrasion using Amnisite BA(R). J Korean Burn Soc. 2012 Jun;15(1):24-29.

14. Fonseca, Walker, Barber, Powers. Oral and Maxillofacial Trauma; 3rd Edition, 2nd volume: Elseiver.

15. Dolan B, Holt L. Accident and Emergency Theory into Practice. Edinburgh, Bailliere Tindall.

16. S, Thomas. Hydrocolloid dressings in the management of acute wounds: a review of the literature. Int Wound J; 2008;5:602-613.

17. Breivik H, et. al. Assessment of pain. British Journal of Anaesthesia 101 (1): 17-24 (2008)

18. Fernandez R, Griffiths R. Wound cleansing, topical antiseptics and wound healing. Int Wound J 2009;6:420-430.

19. Nanda S, Chakraborty S, Ray A, Inamuddin. Healing of cervical necrotising fasciitis using amniotic membrane as dressing material. Natl J Maxillofac Surg. 2011 Jul-Dec; 2(2): 147-151. 